\title{
Commentary: A return trip to MARS: Less may be more when treating mesothelioma
}

\author{
Caitlin M. Griffin, MD, and \\ Victor H. van Berkel, MD, PhD
}

Surgical treatment of mesothelioma continues to be a controversial subject. Extrapleural pneumonectomy (EPP) was considered the standard surgical treatment for malignant pleural mesothelioma from the 1990s until the publication of the Mesothelioma and Radical Surgery (MARS) trial in 2011. ${ }^{1}$ The MARS trial randomized 50 patients to EPP versus no operation after chemotherapy and demonstrated no survival or quality of life benefit for patients undergoing EPP, ${ }^{2}$ with a median survival of 14 months for the surgical group and 19 months for the nonsurgical group. Proponents of EPP argued that the operation still held value in the correct population, although one of the largest datasets published by the late Dr Sugarbaker's group demonstrated a median overall survival after EPP of 18 months, with a 1year survival of $67 \% .^{3}$ These poor results have led to numerous studies exploring less-radical surgical options for the treatment of mesothelioma, including the ongoing MARS2 trial, which is a randomized trial evaluating extended decortication versus no operation, after chemotherapy, for patients with malignant pleural mesothelioma. ${ }^{4}$

In this issue of the Journal, Hasegawa and colleagues ${ }^{5}$ investigate patient outcomes after standard neoadjuvant chemotherapy followed by pleurectomy/decortication (P/ D) in a prospective multi-institutional study. To accomplish this, 24 patients were initially enrolled (with 20 ultimately eligible for inclusion) and underwent 3 cycles of neoadjuvant chemotherapy. Of these 20, 18 proceeded to surgery, with 15 having P/D and 3 requiring intraoperative conversion to EPP. The study had no treatment-related mortality

\footnotetext{
From the Department of Cardiovascular and Thoracic Surgery, University of Louisville School of Medicine, Louisville, Ky.

Disclosures: The authors reported no conflicts of interest.

The Journal policy requires editors and reviewers to disclose conflicts of interest and to decline handling or reviewing manuscripts for which they may have a conflict of interest. The editors and reviewers of this article have no conflicts of interest.

Received for publication July 25, 2021; revisions received July 25, 2021; accepted for publication July 26, 2021; available ahead of print July 29, 2021.

Address for reprints: Victor H. van Berkel, MD, PhD, 201 Abraham Flexner Way, Suite 1200, Louisville, KY 40202 (E-mail: victor.vanberkel@uoflhealth.org).

J Thorac Cardiovasc Surg 2022;163:1948

$0022-5223 / \$ 36.00$

Copyright (C) 2021 by The American Association for Thoracic Surgery

https://doi.org/10.1016/j.jtcvs.2021.07.043
}

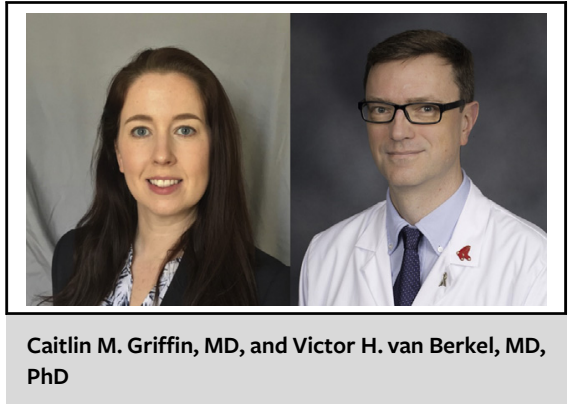

CENTRAL MESSAGE

Pleurectomy and decortication may be reasonable after induction chemotherapy for malignant pleural mesothelioma.

and an overall median survival time (after registration) of 3.45 years for patients undergoing $\mathrm{P} / \mathrm{D}$, a marked improvement over previous studies involving EPP after induction chemotherapy.

One laudable feature of this study is the attempt at standardizing a surgical protocol for a multicenter approach. The authors describe extensive efforts to supply surgeons with scenario descriptions for P/D to EPP conversion, as well as requiring detailed submission of specimen descriptions, surgical photos, and videos. Seminars and written protocols were also used to minimize technique inconsistencies between surgical institutions.

An obvious limitation of the study is the sample size. Although the study is well designed and executed, a total population of 20 patients may not accurately represent results in a larger population. The lack of a control nonsurgical group also makes it difficult to place their results into historical context. The authors make note of this as well as ongoing efforts for a nationwide registry to recruit additional patients.

While it lacks the rigorousness of a randomized control trial, this study provides some hope that the large-scale investigations that are ongoing, including MARS2, will demonstrate that a more conservative surgical approach will result in improved outcomes for patients with this difficult disease. ${ }^{4,6}$

\section{References}

1. Lang-Lazdunski L, Bille A, Lal R, Cane P, McLean E, Landau D, et al. Pleurectomy/decortication is superior to extrapleural pneumonectomy in the multimodality management of patients with malignant pleural mesothelioma. J Thorac Oncol. 2012;7:737-43. 
2. Treasure T, Lang-Lazdunski L, Waller D, Bliss JM, Tan C, Entwisle J, et al. Extrapleural pneumonectomy versus no extra-pleural pneumonectomy for patients with malignant pleural mesothelioma: clinical outcomes of the mesothelioma and radical surgery (MARS) randomised feasibility study. Lancet Oncol. 2011;12: 763-72.

3. Sugarbaker DJ, Richards WG, Bueno R. Extrapleural pneumonectomy in the treatment of epithelioid malignant pleural mesothelioma: novel prognostic implications of combined N1 and N2 nodal involvement based on experience in 529 patients. Ann Surg. 2014;260:577-80; discussion 580-2.

4. Lim E, Darlison L, Edwards J, Elliott D, Fennell DA, Popat S, et al. Mesothelioma and radical surgery 2 (MARS 2): protocol for a multicentre randomised trial comparing (extended) pleurectomy decortication versus no (extended) pleurectomy decortication for patients with malignant pleural mesothelioma. BMJ Open. 2020;10:e038892.

5. Hasegawa S, Yokoi K, Okada M, Tanaka F, Shimokawa M, Daimon T, et al. Neoadjuvant pemetrexed plus cisplatin followed by pleurectomy for malignant pleural mesothelioma. J Thorac Cardiovasc Surg. 2022;163: 1940-7.e5.

6. Shintani Y, Hasegawa S, Takuwa T, Aoe K, Kato K, Fujimoto N, et al. Prospective registry database of patients with malignant mesothelioma: directions for a future Japanese registry-based lung cancer study. J Thorac Dis. 2018;10: 1968-71.
See Article page 1940.

\section{Commentary: Surgical standardization in mesothelioma trials}

\section{Valerie W. Rusch, MD, FACS}

In this issue of the Journal, Hasegawa and colleagues ${ }^{1}$ report the results of a small, multicenter, single-arm, Phase II clinical trial testing the use of neoadjuvant chemotherapy followed by surgical resection in selected patients with malignant pleural mesothelioma (MPM). In essence, this study corroborates the experience reported in earlier trials of similar design showing the feasibility of preoperative chemotherapy followed by surgical resection using extrapleural pneumonectomy (EPP), pleurectomy/decortication (P/D), or extended pleurectomy/decortication (EPD). ${ }^{2,3}$

Perhaps the most important aspect of this study is the rigor of the approach to surgical resection and to documentation of surgical outcomes. Once it became recognized that EPP was associated with a greater morbidity and mortality relative to $\mathrm{P} / \mathrm{D}$ or $\mathrm{EPD}$, with a potential survival benefit seen only in younger patients with node-negative epithelioid

From the Department of Surgery, Memorial Sloan Kettering Cancer Center, New York, NY; and American College of Surgeons, Chicago, Ill.

Dr Rusch's work is supported in part by the National Institutes of Health/National Cancer Institute Cancer Center Support Grant P30 CA008748.

Disclosures: Dr Rusch reported institutional clinical trial funding from Genentech and travel reimbursement from Intuitive Surgical for robotic mentoring.

The Journal policy requires editors and reviewers to disclose conflicts of interest and to decline handling or reviewing manuscripts for which they may have a conflict of interest. The editors and reviewers of this article have no conflicts of interest.

Received for publication Aug 27, 2021; revisions received Aug 27, 2021; accepted for publication Aug 31, 2021; available ahead of print Sept 30, 2021.

Address for reprints: Valerie W. Rusch, MD, FACS, Memorial Sloan-Kettering Cancer Center, 1275 York Ave, New York, NY 10065 (E-mail: ruschv@mskcc.org).

J Thorac Cardiovasc Surg 2022;163:1949-50 $0022-5223 / \$ 36.00$

Copyright (c) 2021 Published by Elsevier Inc. on behalf of The American Association for Thoracic Surgery

https://doi.org/10.1016/j.jtcvs.2021.08.077

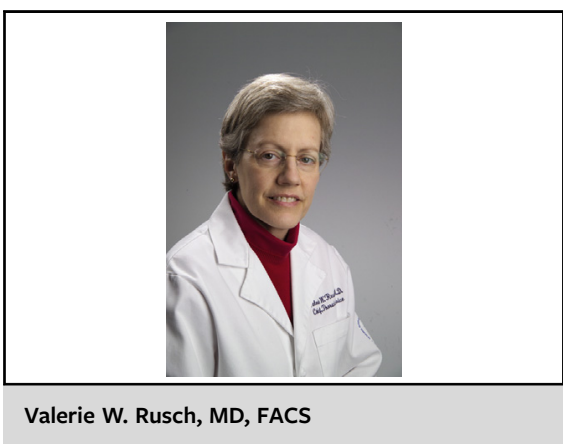

CENTRAL MESSAGE

This study establishes an important bar in terms of technical quality and surgical reporting in clinical trials.

tumors, ${ }^{4,5}$ the thoracic surgical community shifted to performing lung-sparing operations (P/D or EPD). Relative to EPP, these procedures are subject to greater variability in the type of resection because of the heterogeneity in tumor extent that occurs in MPM and a lack of standardization in surgical technique. Since the historical term P/D was too vague a description for lung-sparing operations, the IASLC (International Association for the Study of Lung Cancer) MPM Staging and Prognostic Factors Subcommittee proposed using the term EPD to describe parietal pleurectomy with or without visceral pleural removal (decortication) plus resection of the diaphragm and/or pericardium and retaining the term P/D to describe pleurectomy and decortication only. ${ }^{6}$ This nomenclature was widely adopted but remains insufficient to describe the variations in the extent of lung-sparing operations which in turn influence surgical risk and outcomes, particularly in the context of multimodality treatment. The IASLC MPM subcommittee, 